\title{
Luteolin Promotes Apoptosis of Endometriotic Cells and Inhibits the Alternative Activation of Endometriosis-Associated Macrophages
}

\author{
Jeong-Hwa Woo ${ }^{1}$, Dae Sik Jang ${ }^{1,2, *}$, and Jung-Hye Choi ${ }^{1,2, *}$ \\ ${ }^{1}$ College of Pharmacy, Kyung Hee University, Seoul 02447, \\ ${ }^{2}$ Department of Life and Nanopharmaceutical Sciences, Kyung Hee University, Seoul 02447, Republic of Korea
}

\begin{abstract}
Luteolin, a flavonoid present in several fruits, vegetables, nuts, and herbs reportedly exhibits anti-cancer and anti-inflammatory properties. However, the effect of luteolin on endometriosis, a painful condition characterized by the ectopic growth of endometrial tissue and pelvic inflammation, remains elusive. Herein, we observed that luteolin inhibited cell growth and induced apoptosis of $12 Z$ human endometriotic cells by activating caspase-3, -8 , and -9 . Additionally, luteolin significantly inhibited the expression of key chemokines, C-C motif chemokine ligand 2 (CCL2) and CCL5, required for monocyte/macrophage influx at endometriotic sites. In macrophages stimulated by endometriotic cells, luteolin treatment suppressed the intracellular expression of M2 markers and endometriosis-promoting factors. Collectively, our data suggest that luteolin exerts anti-endometriotic effects by stimulating endometriotic cell apoptosis and hindering the alternative activation of macrophages.
\end{abstract}

Key Words: Luteolin, Endometriosis, Macrophages, Apoptosis, M2 polarization

\section{INTRODUCTION}

Endometriosis is a common gynecological disease characterized by endometrial tissue growth outside the uterus, owing to increased cellular proliferation or decreased apoptosis in response to appropriate stimuli. Globally, endometriosis reportedly affects $5 \%-20 \%$ of women of reproductive age, with patients generally experiencing non-cyclical pelvic pain, dyspareunia, dysmenorrhea, and subfertility. Based on accumulated evidence, apoptosis helps maintain cellular homeostasis by promoting the removal of senescent cells from the functional layer of the endometrial lining during menstrual and late secretory phases of the cycle (Herington et al., 2011). In patients with endometriosis, the apoptotic cell population in sloughed endometrial cells is found to be markedly decreased (Khazaei et al., 2016), suggesting that women who develop endometriosis have an increased number of surviving cells entering the peritoneal cavity. Macrophage recruitment and activation reportedly play a key role in endometriosis (Bondza et al., 2009). In patients with endometriosis, macrophage populations are significantly increased in peritoneal lesions (Hever et al., 2007). Notably, ectopic sites show increased macrophage activation, along with increased levels of synthesis and secretion of various pro-inflammatory mediators, such as interleukins (Wu and Ho, 2003), other cytokines (Taylor et al., 1997), and angiogenic factors (Kyama et al., 2003), when compared with those in the peritoneal fluid or peritoneum of normal controls (Kyama et al., 2003; Siristatidis et al., 2006). These findings suggest that agents that induce endometriotic cell apoptosis and inhibit macrophage activation could be developed as potential therapeutic candidates for endometriosis.

Luteolin (3,4,5,7-tetrahydroxyflavone) is a natural flavonoid abundant in several food sources, including fruits, vegetables, nuts, and herbs (Lopez-Lazaro, 2009; Bhagwat et al., 2011). Dietary sources rich in luteolin include carrot, broccoli, parsley, celery, olives, thyme, and clove. Previous studies have revealed that luteolin possesses numerous pharmacological properties, including antioxidant, anti-inflammatory, anti-allergic, and anti-tumor activities (Lopez-Lazaro, 2009; Jang et al., 2010; Nazari et al., 2013; Si et al., 2014). For example, luteolin reportedly attenuates oxidative damage and lipid peroxidation in peripheral nerves of patients with diabetes (Li et al., 2015). Additionally, luteolin effectively inhibits the expression of lipopolysaccharide-induced pro-inflammatory mediators in vitro

\section{Open Access https://doi.org/10.4062/biomolther.2021.045}

This is an Open Access article distributed under the terms of the Creative Commons Attribution Non-Commercial License (http://creativecommons.org/licenses/by-nc/4.0/) which permits unrestricted non-commercial use, distribution, and reproduction in any medium, provided the original work is properly cited.
Received Mar 7, 2021 Revised Apr 4, 2021 Accepted Apr 19, 2021 Published Online May 20, 2021

\section{${ }^{*}$ Corresponding Authors}

E-mail: dsjang@khu.ac.kr (Jang DS), jchoi@khu.ac.kr (Choi JH) Tel: +82-2-961-0719 (Jang DS), +82-2-961-2172 (Choi JH) Fax: +82-2-961-9580 (Jang DS), +82-2-961-9580 (Choi JH) 
and reduces leukocyte infiltration in vivo (Kotanidou et al., 2002). Luteolin inhibits the proliferation of human glioblastoma cells by inducing cell cycle arrest and apoptosis (Tsai et al., 2013). Moreover, luteolin treatment has been shown to reduce the number of eosinophils, neutrophils, and lymphocytes, as well as the levels of T helper 2 cytokines, in a murine model of allergic asthma and rhinitis (Jang et al., 2017). However, the effect of luteolin on endometriosis needs to be clarified. Therefore, in the present study, we evaluated the anti-endometriotic effects of luteolin using $12 Z$ human endometriotic cells and macrophages stimulated by endometriotic cells.

\section{MATERIALS AND METHODS}

\section{Materials}

Luteolin was isolated from the ethyl acetate (EtOAc) fraction of the $70 \%$ EtOH extract flower buds of Syzygium aromaticum as described in our previous study (Ryu et al., 2016). DMEM media and RPMI 1640 media, fetal bovine serum (FBS), penicillin, and streptomycin were purchased from Life Technologies, Inc (Grand Island, NY, USA). Propidium iodide $(\mathrm{PI})$, phorbol myristate acetate (PMA), 2-mercaptoethanol, and caspase inhibitors were purchased from Sigma Aldrich Co (St. Louis, MO, USA). MTT (3-[4,5-Dimethylthiazol-2-yl]-2,5diphenyltetrazolium bromide) was procured from Molecular Probes, Inc (Eugene, OR, USA). Annexin V-fluorescein isothiocyanate (FITC) and phenylmethylsulfonylfluoride (PMSF) were obtained from BD Biosciences (San Diego, CA, USA). The Enhanced chemiluminescence $(E C L)$ detection reagent kit was purchased from AbClon Inc (Seoul, Korea). Caspase-8 antibody was purchased from Santa Cruz Biotechnology (Santa Cruz, CA, USA). Anti-caspase- 3 and -9 antibodies were procured from Cell Signaling Technology (Beverly, MA, USA). Protein lysis buffer and Easy Blue kit for RNA extraction were supplied from Intron Biotechnology (Seoul, Korea). Protease inhibitor cocktail was purchased from Boehringer Mannheim (Mannheim, Germany). SYBR Premix Ex Taq was supplied from TaKaRa (Tokyo, Japan). First-Strand cDNA synthesis kit was procured from Amersham Pharmacia Biotech (Oakville, ON, Canada).

\section{Cell culture}

Human endometriotic $12 Z$ cells were generously gifted by Dr. Starzinski-Powitz (Johann-Wolfgang-Goethe-Universitaet, Frankfurt, Germany) and were maintained at $37^{\circ} \mathrm{C}$ in a containing $5 \% \mathrm{CO}_{2}$ and cultured in DMEM containing $5 \%(\mathrm{v} / \mathrm{v})$ fetal bovine serum, $100 \mathrm{U} / \mathrm{ml}$ penicillin, and $100 \mu \mathrm{g} / \mathrm{mL}$ streptomycin sulfate. The cells were treated with vehicle (DMSO) or luteolin. The human monocyte THP-1 cells were purchased from American Type Culture Collection (ATCC, Manassas, VA, USA) and cultured in RPMI 1640 containing FBS 10\% (v/v), streptomycin sulfate $(100 \mu \mathrm{g} / \mathrm{mL})$, penicillin $(100 \mathrm{U} / \mathrm{mL})$, and 2-mercaptoethanol ( $0.05 \mathrm{mM})$. THP-1 cells were differentiated into macrophages with $100 \mathrm{nM}$ phorbol 12-myristate 13-acetate (PMA) for $24 \mathrm{~h}$. To prepare the endometriosis-associated macrophages (EAMs), the macrophages were stimulated with conditioned medium (CM) containing various soluble factors derived from endometriotic $12 Z$ cells. The $12 Z$ cells $\left(1 \times 10^{6}\right)$ with $3 \mathrm{~mL}$ complete medium were seeded and cultured in 60 $\mathrm{mm}$ dish. After $48 \mathrm{~h}$, the medium of the cultures was harvested and used for stimulation of macrophages.

\section{Cell viability}

$12 Z$ cells $\left(1 \times 10^{5}\right)$ were seeded into each well of a 96 -well plates and allowed for incubation overnight. After $48 \mathrm{~h}$ treatment, $25 \mu \mathrm{L}$ of a $0.5 \mathrm{mg} / \mathrm{mL}$ MTT solution was added into each well and the cells were further incubated at $37^{\circ} \mathrm{C}$ for $4 \mathrm{~h}$. Thereafter the media was aspirated and dimethyl sulfoxide (DMSO) was added to dissolve the formazan blue. Absorbance of the samples was determined at a wavelength of $540 \mathrm{~nm}$ by Spectra Max (Molecular Devices, Sunnyvale, CA, USA).

\section{Apoptosis analysis}

Cells were treated with luteolin for $24 \mathrm{~h}$. After collection, the cells were washed in ice-cold PBS. Subsequently, the cells were centrifuged at 1,000 rpm for $10 \mathrm{~min}$ and washed twice with PBS. The cells were resuspended with a binding buffer (0.1 M Hepes/NaOH pH 7.4, 25 mM CaCl $2,1.4 \mathrm{M} \mathrm{NaCl}$ ), containing PI and Annexin V- FITC, and stored in the dark at room temperature for $20 \mathrm{~min}$. Apoptosis of cells analyzed by Guava easyCyte flow cytometry system (Millipore Corporation, Billerica, MA, USA).

\section{Western blot}

Lysates from cells were electrophoresed on $10 \%$ sodium dodecyl sulfate polyacrylamide gel electrophoresis (SDSPAGE) gel and transferred to the polyvinylidene fluoride (PVDF) membranes at $300 \mathrm{~mA}$ for $1 \mathrm{~h}$. The membranes were blocked in 5\% skimmed milk/TBST (Tris-buffered saline buffer containing $0.1 \%$ Tween-20) for $30 \mathrm{~min}$, followed by incubated with primary antibodies using TBST with $3 \%$ skimmed milk. Antibodies used include: caspase-3 $(1: 1,000)$, caspase-8 $(1: 1,000)$, caspase- $9(1: 1,000)$, and $\beta$-actin $(1: 1,000)$. The washed membranes were probed with horseradish peroxidase-conjugated secondary antibody $(1: 1,000)$ in TBST with $3 \%$ skimmed milk. The membranes were washed again three times in TBST buffer and incubated with ECL solution for 1 min. Immunoreactivity for the transferred proteins was visualized and analyzed by Image Quant LAS-4000 (Fujifilm Life science, Tokyo, Japan).

\section{Quantitative real-time PCR}

According to the manufacture's protocol, total RNA was isolated from $12 Z$ cells and macrophages using Easy Blue reagent (Invitrogen, San Diego, CA, USA). After total RNA was reverse transcribed into the cDNA using first-strand CDNA synthesis kit (Amersham Pharmacia Biotech), SYBR Premix Ex Taq $^{\mathrm{TM}}$ Kit and Thermal Cycler Dice Real-Time PCR System (TaKaRa) were used for the reverse-transcription (RT)PCR. The primers pairs used for real-time RT-PCR reactions (Bioneer, Seoul, Korea) are as follow (forward and reverse): human CCL5, 5'-CCTCATTGCTAGGCCCTCT-3' and 5'-GGTGTGGTGTCCCGAGGAAT-3'; human CCL2, 5'-GCTCATAGCAGCCACCTTCA-3' and 5'-GGACACTTGCTGCTGGTGAT-3'; humanCD206,5'-ACCTCACAAGTATCCACACCATC-3' and 5'-CTTTCATCACCACACAATCCTC-3'; human Trem-2, 5'-TTGCCCCTATGACTCCATGA-3' and 5'-CGCAGCGTAATGGTGAGAGT-3'; human MMP-9, 5'-CGATGACGAGTTGTGGTCCC-3' and 5'-TCGTAGTTGGCCGTGGTACT-3'; human MMP (matrix metalloproteinase)-2, 5'-ACCGCGACAAGAAGTATGGC-3' and 5'-CCACTTGCGGTCATCATCGT-3'; human IL-10, 5'-GACCAGCTGGACAACATACTGCTAA-3' and 5'-GAT AAGGCTTGGCAACCCAAGTAA-3'; human VEGF (vascular endothelial growth factor), 5'-ATGGCAGAAGGAGGAGG 
GCA-3' and 5'-ATCGCATCAGGGGCACACAG-3'; human GA PDH, 5'-GAGTCAACGGATTTGGTCGT-3' and 5'-TTGATTTT GGAGGGATCTCG-3'. To analyze the relative mRNA levels of target gene, the average cycle threshold $(\mathrm{Ct})$ value of each triplicate reaction was normalized relative to that of an internal control, GAPDH. Melting curve analysis was performed to verify the presence of gene-specific peak and the absence of primer dimer.

\section{Statistical analysis}

Statistical analysis for comparison was performed using the One-way ANOVA or Student's $t$-test. Statistical significance was accepted at $p$-value $<0.05$. Data were expressed as mean \pm SD of at least three independent experiments.

\section{RESULTS}

\section{Luteolin inhibits cell growth via apoptosis induction in human endometriotic cells}

Endometriosis is characterized by the ectopic growth of endometrial tissues. In the present study, we investigated the effect of luteolin (Fig. 1A) on the growth of $12 Z$ human endometriotic cells using the MTT 3-(4,5-dimethylthiazol-2-yl)-2,5diphenyltetrazolium bromide) assay. Luteolin significantly inhibited cell growth in $12 Z$ cells in a dose-dependent manner (Fig. 1B), prompting us to further explore the molecular mechanism underlying this effect. To investigate whether luteolin-

A<smiles>O=c1cc(-c2ccc(O)c(O)c2)oc2cc(O)cc(O)c12</smiles>

Luteolin

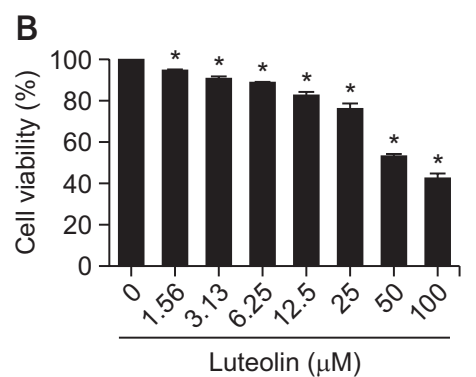

Fig. 1. Effect of luteolin on cell viability in human endometriotic cells. (A) Chemical structure of luteolin (B) $12 Z$ cells were treated with the indicated concentration $(1.56,3.13,6.25,12.5,25,50$, and $100 \mu \mathrm{M}$ ) of luteolin for $48 \mathrm{~h}$. MTT assay was performed to determine the cell viability after luteolin treatment. ${ }^{*} p<0.05$ as compared with the control group. induced cell death is associated with the induction of apoptotic cell death, Annexin V-FITC staining was performed. As shown in Fig. 2, luteolin markedly enhanced the population of apoptotic Annexin V-FITC-positive cells in a dose-dependent manner, indicating that luteolin induces apoptotic cell death in human endometriotic cells.

\section{Luteolin promotes apoptosis through caspase activation in human endometriotic cells}

Next, we investigated whether luteolin-induced apoptosis was caspase-dependent. Western blotting was performed to evaluate the activation of initiator caspases (caspase- 8 and caspase-9) and an effector caspase (caspase-3) indicated by the density of pro-caspases. As shown in Fig. 3A, luteolin dose-dependently and markedly decreased the levels of procaspase $-3,-8$, and -9 in $12 Z$ cells. Various caspase inhibitors were then employed to further confirm the involvement of caspases in luteolin-induced cell death (Fig. 3B). Pretreatment with Z-VAD-FMK (a broad caspase inhibitor), Z-DEVD-FMK (caspase-3 inhibitor), Z-IEVD-FMK (caspase-8 inhibitor), and Z-LEHD-FMK (caspase-9 inhibitor) markedly reduced the inhibitory effect of luteolin on cell growth. These findings indicate the involvement of the caspase-dependent pathway in luteolin-induced apoptosis in human endometriotic cells.

\section{Luteolin downregulates the expression of the chemokines CCL2 and CCL5 in human endometriotic cells}

The increased production of chemokines, such as C-C motif chemokine ligand 2 (CCL2) and CCL5, by endometriotic tissues, has been postulated to promote a pro-inflammatory environment that fuels disease pathogenesis by inducing macrophage recruitment to endometriotic lesions (Hornung et al., 2001; Lebovic et al., 2001, 2004). We examined the effect of luteolin on the expression of CCL2 and CCL5 in human endometriotic cells. Luteolin significantly suppressed the intracellular mRNA levels of CCL2 and CCL5 in $12 Z$ cells (Fig. 4). This finding suggests that luteolin may inhibit macrophage recruitment to the endometriotic lesion by downregulating the expression of the chemokines CCL2 and CCL5, thereby suppressing their secretion by endometriotic cells.

\section{Luteolin downregulates the expression of M2 phenotype markers and endometriosis-promoting factors in macrophages stimulated by human endometriotic cells}

Reportedly, macrophages are recruited to endometriotic lesions, where they undergo alternative activation (M2 polar-
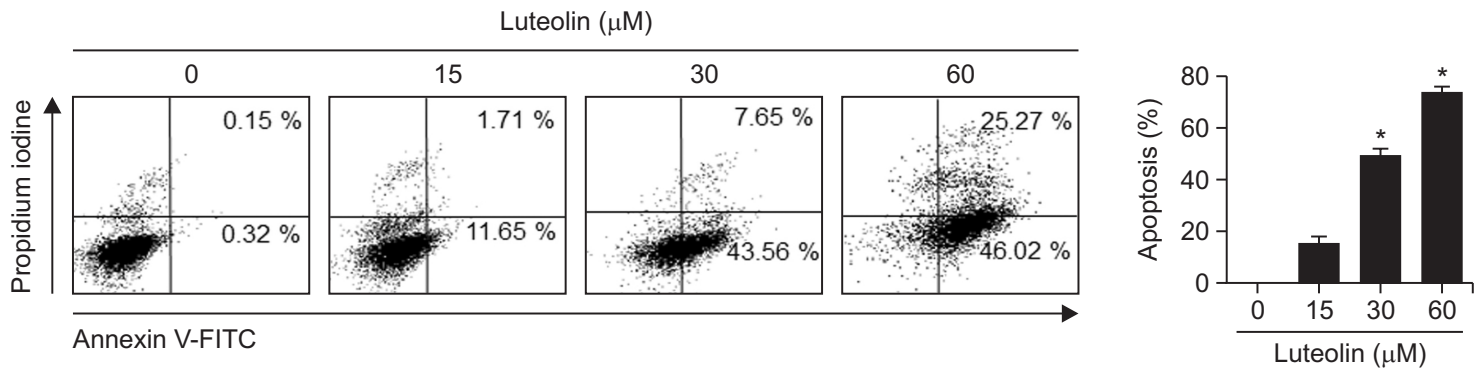

Fig. 2. Annexin V-FITC staining assay for analysis of the apoptotic cell death in human endometriotic cells. $12 Z$ cells were treated with luteolin $(15,30$, and $60 \mu \mathrm{M})$ for $48 \mathrm{~h}$ and then stained with Annexin V-FITC. The translocation of phosphatidylserine was detected by flow cytometry. The graph indicates the percentages of apoptosis. ${ }^{*} p<0.05$ as compared with the control group. 
A

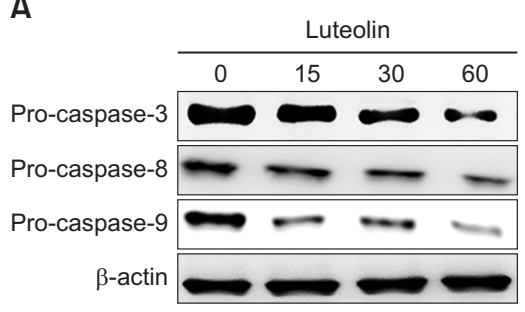

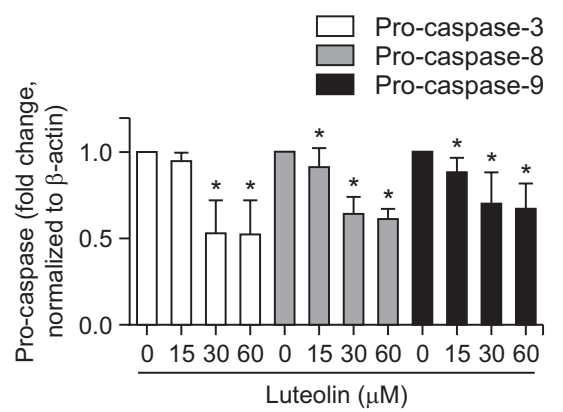

B

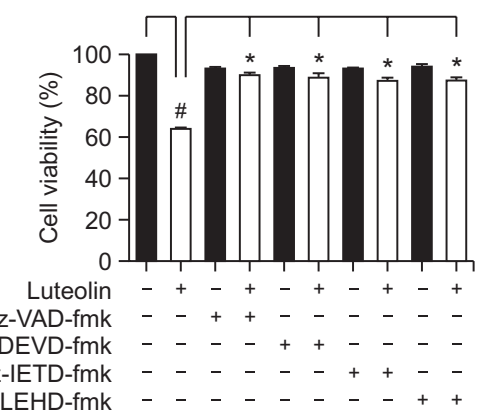

Fig. 3. Involvement of caspases in luteolin-induced cell death in human endometriotic cells. (A) $12 Z$ cells were treated with luteolin (15, 30, and $60 \mu \mathrm{M})$ for $48 \mathrm{~h}$. Pro-caspase-3, -8 , and -9 levels were determined by Western blot assay. $\beta$-Actin was used as a control. ${ }^{*} p<0.05$ as compared the control group. (B) $12 \mathrm{Z}$ cells were pretreated with broad caspase inhibitor z-VAD-FMK (50 $\mu \mathrm{M})$, caspase-3 inhibitor z-DEVDFMK $(50 \mu \mathrm{M})$, caspase-8 inhibitor z-IETD FMK $(50 \mu \mathrm{M})$, and caspase-9 inhibitor z-LEHD-FMK $(50 \mu \mathrm{M})$ for 30 min, and then treated with luteolin $(30 \mu \mathrm{M})$ for $48 \mathrm{~h}$. MTT assay wasperformed to determine the cell viability after luteolin treatment. ${ }^{*} p<0.05$ as compared with the control group, and ${ }^{*} p<0.05$ as compared with the luteolin-treated group.
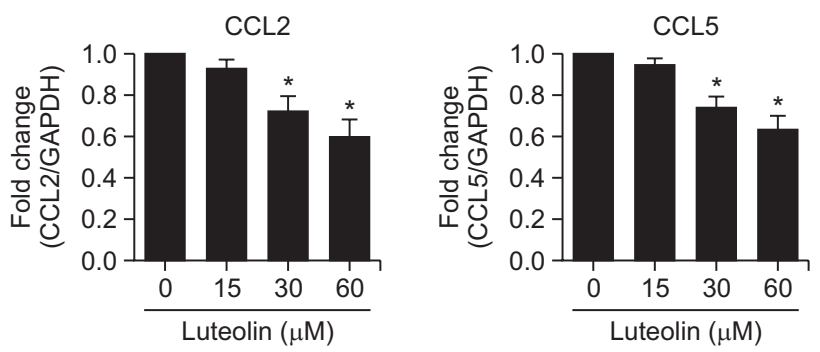

Fig. 4. Effect of luteolin on the expression of CCL2 and CCL5 in human endometriotic cells. $12 \mathrm{Z}$ cells were treated with luteolin $(15,30$, and $60 \mu \mathrm{M})$ for $48 \mathrm{~h}$. Real-time RT-PCR was performed to measure the mRNA levels of CCL2 and CCL 5 in $12 Z$ cells. GAP. $\mathrm{DH}$ was used as an internal control. ${ }^{*} p<0.05$ as compared with the control group.

ization) (Capobianco and Rovere-Querini, 2013). Therefore, we investigated the effect of luteolin on the M2 polarization of macrophages. Macrophages were stimulated by conditioned medium containing various soluble factors (cytokines, chemokines, and growth factors) derived from endometriotic $12 Z$ cells and the so-called endometriosis-associated macrophages (EAMs), revealing increased intracellular expression of the CD206 and Trem-2 M2 phenotype markers when compared with that in control macrophages (Fig. 5). Treatment with luteolin significantly reduced the mRNA expression of CD206 and Trem-2 in EAMs. Notably, EAMs are known to produce various endometriosis-promoting factors, such as matrix metalloproteinase (MMP)-2/9, interleukin (IL)-10, and vascular endothelial growth factor (VEGF) (Woo et al., 2017). As shown in Fig. 6, luteolin significantly inhibited the intracellular expression of IL-10, VEGF, and MMP-2/-9 in EAMs. Our findings suggested that luteolin suppressed macrophage activation, which is known to promote endometriosis progression.

\section{DISCUSSION}

In recent years, natural compounds commonly found to occur in food have been widely researched to prevent and treat various gynecological disorders, including endometriosis (Varma et al., 2004). Numerous studies have suggested
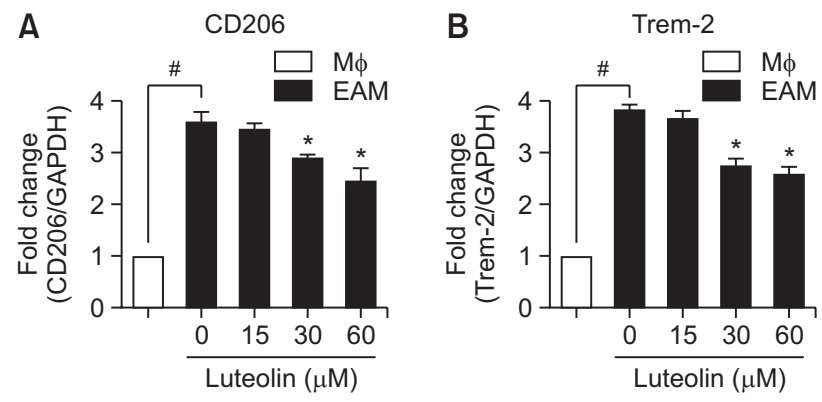

Fig. 5. Effect of luteolin on the expression of CD206 and Trem-2 in macrophages stimulated by conditioned medium from human endometriotic $12 Z$ cells (EAMs). THP- 1 macrophages were stimulated with conditioned medium from $12 Z$ cells for $24 \mathrm{~h}$. The activated macrophages (EAMs) were treated with luteolin $(15,30$, and 60 $\mu \mathrm{M})$ for $24 \mathrm{~h}$. Real-time RT-PCR was performed to measure the mRNA levels of CD206 (A) and Trem-2 (B) in macrophages. GAP$\mathrm{DH}$ was used as an internal control. ${ }^{\#} p<0.05$ as compared with macrophage control, and ${ }^{*} p<0.05$ as compared with EAMs control.

that resveratrol, a phytoalexin polyphenol present in several dietary sources, including soy, grapes, and peanuts, possesses anti-endometriotic properties, which are mediated by the inhibition of inflammation and cell proliferation as well as apoptosis induction (Bruner-Tran et al., 2011; Amaya et al., 2014; Dull et al., 2019). Apigenin, a flavonoid in vegetables and fruits, such as onions, oranges, and parsley, reportedly induces apoptosis and cell cycle arrest in human endometriotic cells (Park et al., 2018). Another flavonoid, puerarin, has been shown to inhibit the invasion and vascularization of endometriotic tissue in rat endometriosis models (Chen et al., 2011; Wang et al., 2011). Notably, luteolin, a common dietary flavonoid, induces cell cycle arrest and apoptosis in human endocervical cells and a mouse endometriosis model (Park et al., 2019). Herein, we investigated the effect of luteolin on the growth of human endometriotic cells and the alternative activation of macrophages stimulated by endometriotic cells.

The growth of endometriotic cells plays a central role in the development of endometriotic lesions (Di Paola et al., 2016). Indeed, increased cell growth can be observed in the eutopic and ectopic endometrial tissues of patients with endometriosis, while apoptotic events are reduced in endometriotic le- 

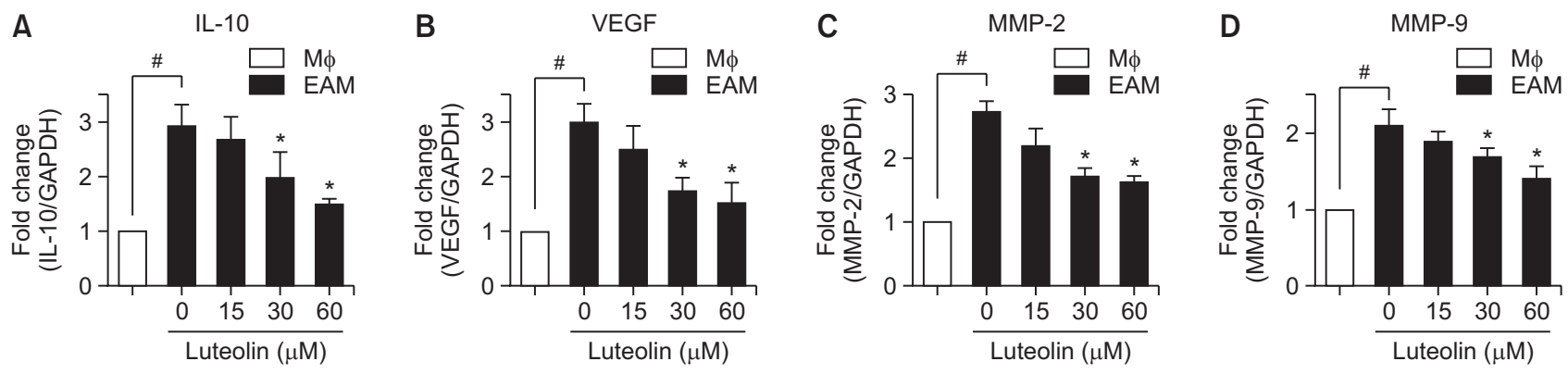

Fig. 6. Effect of luteolin on the expression and production of IL-10, VEGF, MMP-2, and MMP-9 in macrophages stimulated by conditioned medium from human endometriotic $12 \mathrm{Z}$ cells. THP-1 macrophages were stimulated with conditioned medium from $12 \mathrm{Z}$ cells for $24 \mathrm{~h}$. The activated macrophages (EAMs) were treated with luteolin $(15,30$, and $60 \mu \mathrm{M})$ for $24 \mathrm{~h}$. Real-time RT-PCR was performed to measure the mRNA levels of IL-10, VEGF, MMP-2, and MMP-9 in macrophages. GAPDH was used as an internal control. ${ }^{\#} p<0.05$ as compared with macrophage control, and ${ }^{*} p<0.05$ as compared with EAMs control.

sions (Wingfield et al., 1995). These findings suggest that one possible strategy to treat endometriosis is to inhibit cell growth and induce apoptosis of endometriotic cells. Several studies have suggested that luteolin may inhibit cell growth by stimulating cell cycle arrest (Park et al., 2014), autophagy (Cao et al., 2017), and apoptosis in various cancer cells (Wang et al., 2012). In the present study, we observed that luteolin significantly induced apoptotic cell death in human endometriotic cells. The caspase family is known to function as a key mediator of apoptotic cell death. The intrinsic or extrinsic signaling pathway activates caspase-dependent apoptotic cell death. Activated initiator caspases, including caspase-8 and caspase-9, can induce the activation of effector caspases, such as caspase- 3 . The activation of caspase- 8 is related to the extrinsic (death receptor-mediated) pathway, whereas caspase- 9 is an initiator caspase for the mitochondria-mediated intrinsic pathway. In the present study, luteolin stimulated caspase-8, caspase-9, and caspase-3 activation in endometriotic cells. Furthermore, specific inhibitors of caspase-8 and caspase-9 (Z-IEVD-FMK and Z-LEHD-FMK, respectively) reversed luteolin-induced apoptosis in $12 Z$ cells, indicating that luteolin induces caspase-dependent apoptotic cell death in human endometriotic cells.

The levels of CCL2 and CCL5, which play vital roles in macrophage recruitment, are known to be significantly increased in the peritoneal fluid of women with endometriosis (Arici et al., 1997). In patients with endometriosis and animal models, macrophages can be alternatively activated in inflammatory peritoneal lesions and fluid, respectively (Bacci et al., 2009). These macrophages show high expression of M2 markers, such as CD163 and CD206. Interestingly, M2 macrophages promote the growth of endometriotic lesions (Capobianco and Rovere-Querini, 2013). In addition, the M2 polarization of macrophages facilitates invasion and fibrogenesis in endometriosis (Duan et al., 2018). Moreover, M2 macrophages have been shown to secrete several factors, including VEGF, IL-10, and MMPs, which play a role in the development and progression of endometriosis (Villalta et al., 2011; Traves et al., 2012). IL-10 is highly expressed in activated macrophages (Wu and Ho, 2003) and is known to suppress T-cell function (e.g., downregulation of the Thelper 1 type immune response) in the peritoneal fluid of patients with endometriosis (Rana et al., 1996). Activated macrophages release several MMPs, including MMP-2 and MMP-9, which are type IV collagenases that effectively degrade principal components of basement membranes. MMP-2 and MMP-9 are thought to be involved in the pathogenesis of endometriosis and cancer, especially in the context of migration and invasion (Cominelli et al., 2014). Several studies have reported increased expression levels of MMP-2 and MMP-9 in endometriotic tissues (Wenzl and Heinzl, 1998; Chung et al., 2001). Furthermore, M2 macrophages express proangiogenic factors, such as VEGF (Li et al., 2016) with elevated VEGF levels detected in the peritoneal fluid of women with endometriosis (McLaren et al., 1996b). McLaren et al. (1996a) have reported that VEGF is synthesized and secreted by activated peritoneal fluid macrophages and that macrophage-derived VEGF stimulates angiogenesis in women with endometriosis. Collectively, these findings suggest that inhibition of macrophage recruitment and alternative activation can be a potential strategy to treat endometriosis. In the present study, we demonstrated that luteolin inhibited the expression of chemoattractants CCL2 and CCL5 in human endometriotic cells. Luteolin also suppressed M2 polarization and pro-endometriotic factor expression in macrophages stimulated by endometriotic cells. Notably, some dietary flavonoids have been suggested to exert anti-cancer effects by suppressing macrophage recruitment and M2 macrophage activation. For example, isoliquiritigenin markedly suppressed colitis-induced tumorigenesis by inhibiting M2 macrophage polarization (Zhao et al., 2014). In addition, puerarin inhibits tumor growth by inhibiting M2 macrophages in a non-small cell lung cancer xenograft murine model (Kang et al., 2017). We demonstrated that luteolin inhibits the expression of CCL2 and CCL5, major determining factors of macrophage recruitment at endometriosis sites, thus resulting in the suppression of alternatively activated macrophages. Additionally, luteolin suppressed the expression of M2 markers (CD206 and Trem2) and endometriosis-promoting cytokines (IL-10 and VEGF) and metalloproteinase enzymes (MMP-2 and MMP-9) in EAMs. This is the first study to report the inhibitory effect of luteolin on M2 macrophages in endometriosis.

Overall, these data indicate that luteolin stimulates apoptosis in human endometriotic cells and suppresses the activation of EAMs, implying that the anti-inflammatory properties of luteolin are associated with its inhibitory effect on EAMs and endometriotic cells. 


\section{CONFLICT OF INTEREST}

Authors have nothing to declare.

\section{ACKNOWLEDGMENTS}

This work was supported by a grant the National Research Foundation of Korea (NRF) (No. NRF-2017R1A6A3A11034320 and NRF-2020R111A1A01075620).

\section{REFERENCES}

Amaya, S. C., Savaris, R. F., Filipovic, C. J., Wise, J. D., Hestermann, E., Young, S. L. and Lessey, B. A. (2014) Resveratrol and endometrium: a closer look at an active ingredient of red wine using in vivo and in vitro models. Reprod. Sci. 21, 1362-1369.

Arici, A., Oral, E., Attar, E., Tazuke, S. I. and Olive, D. L. (1997) Monocyte chemotactic protein-1 concentration in peritoneal fluid of women with endometriosis and its modulation of expression in mesothelial cells. Fertil. Steril. 67, 1065-1072.

Bacci, M., Capobianco, A., Monno, A., Cottone, L., Di Puppo, F., Camisa, B., Mariani, M., Brignole, C., Ponzoni, M., Ferrari, S., PaninaBordignon, P., Manfredi, A. A. and Rovere-Querini, P. (2009) Macrophages are alternatively activated in patients with endometriosis and required for growth and vascularization of lesions in a mouse model of disease. Am. J. Pathol. 175, 547-556.

Bondza, P. K., Maheux, R. and Akoum, A. (2009) Insights into endometriosis-associated endometrial dysfunctions: a review. Front. Biosci. (Elite Ed.) 1, 415-428.

Bruner-Tran, K. L., Osteen, K. G., Taylor, H. S., Sokalska, A., Haines, K. and Duleba, A. J. (2011) Resveratrol inhibits development of experimental endometriosis in vivo and reduces endometrial stromal cell invasiveness in vitro. Biol. Reprod. 84, 106-112.

Cao, Z., Zhang, H., Cai, X., Fang, W., Chai, D., Wen, Y., Chen, H., Chu, F. and Zhang, Y. (2017) Luteolin promotes cell apoptosis by inducing autophagy in hepatocellular carcinoma. Cell. Physiol. Biochem. 43, 1803-1812.

Capobianco, A. and Rovere-Querini, P. (2013) Endometriosis, a disease of the macrophage. Front. Immunol. 4, 9.

Chen, Y., Chen, C., Shi, S., Han, J., Wang, J., Hu, J., Liu, Y., Cai, Z. and Yu, C. (2011) Endometriotic implants regress in rat models treated with puerarin by decreasing estradiol level. Reprod. Sci. 18, 886-891.

Chung, H. W., Wen, Y., Chun, S. H., Nezhat, C., Woo, B. H. and Lake Polan, M. (2001) Matrix metalloproteinase- 9 and tissue inhibitor of metalloproteinase-3 mRNA expression in ectopic and eutopic endometrium in women with endometriosis: a rationale for endometriotic invasiveness. Fertil. Steril. 75, 152-159.

Cominelli, A., Gaide Chevronnay, H. P., Lemoine, P., Courtoy, P. J., Marbaix, E. and Henriet, P. (2014) Matrix metalloproteinase-27 is expressed in CD163+/CD206+ M2 macrophages in the cycling human endometrium and in superficial endometriotic lesions. Mol. Hum. Reprod. 20, 767-775.

Di Paola, R., Fusco, R., Gugliandolo, E., Crupi, R., Evangelista, M., Granese, R. and Cuzzocrea, S. (2016) Co-micronized palmitoylethanolamide/polydatin treatment causes endometriotic lesion regression in a rodent model of surgically induced endometriosis. Front. Pharmacol. 7, 382.

Duan, J., Liu, X., Wang, H. and Guo, S. W. (2018) The M2a macrophage subset may be critically involved in the fibrogenesis of endometriosis in mice. Reprod. Biomed. Online 37, 254-268.

Dull, A. M., Moga, M. A., Dimienescu, O. G., Sechel, G., Burtea, V. and Anastasiu, C. V. (2019) Therapeutic approaches of resveratrol on endometriosis via anti-inflammatory and anti-angiogenic pathways. Molecules 24, 667.

Herington, J. L., Bruner-Tran, K. L., Lucas, J. A. and Osteen, K. G. (2011) Immune interactions in endometriosis. Expert Rev. Clin. Immunol. 7, 611-626.
Hever, A., Roth, R. B., Hevezi, P., Marin, M. E., Acosta, J. A., Acosta, H., Rojas, J., Herrera, R., Grigoriadis, D., White, E., Conlon, P. J., Maki, R. A. and Zlotnik, A. (2007) Human endometriosis is associated with plasma cells and overexpression of B lymphocyte stimulator. Proc. Natl. Acad. Sci. U.S.A. 104, 12451-12456.

Hornung, D., Bentzien, F., Wallwiener, D., Kiesel, L. and Taylor, R. N. (2001) Chemokine bioactivity of RANTES in endometriotic and normal endometrial stromal cells and peritoneal fluid. Mol. Hum. Reprod. 7, 163-168.

Jang, S., Dilger, R. N. and Johnson, R. W. (2010) Luteolin inhibits microglia and alters hippocampal-dependent spatial working memory in aged mice. J. Nutr. 140, 1892-1898.

Jang, T. Y., Jung, A. Y., Kyung, T. S., Kim, D. Y., Hwang, J. H. and Kim, Y. H. (2017) Anti-allergic effect of luteolin in mice with allergic asthma and rhinitis. Cent. Eur. J. Immunol. 42, 24-29.

Kang, H., Zhang, J., Wang, B., Liu, M., Zhao, J., Yang, M. and Li, Y. (2017) Puerarin inhibits M2 polarization and metastasis of tumorassociated macrophages from NSCLC xenograft model via inactivating MEK/ERK 1/2 pathway. Int. J. Oncol. 50, 545-554.

Khazaei, M. R., Rashidi, Z., Chobsaz, F. and Khazaei, M. (2016) Apoptosis induction of human endometriotic epithelial and stromal cells by noscapine. Iran. J. Basic Med. Sci. 19, 940-945.

Kotanidou, A., Xagorari, A., Bagli, E., Kitsanta, P., Fotsis, T., Papapetropoulos, A. and Roussos, C. (2002) Luteolin reduces lipopolysaccharide-induced lethal toxicity and expression of proinflammatory molecules in mice. Am. J. Respir. Crit. Care Med. 165, 818-823.

Kyama, C. M., Debrock, S., Mwenda, J. M. and D'Hooghe, T. M. (2003) Potential involvement of the immune system in the development of endometriosis. Reprod. Biol. Endocrinol. 1, 123.

Lebovic, D. I., Chao, V. A. and Taylor, R. N. (2004) Peritoneal macrophages induce RANTES (regulated on activation, normal $T$ cell expressed and secreted) chemokine gene transcription in endometrial stromal cells. J. Clin. Endocrinol. Metab. 89, 1397-1401.

Lebovic, D. I., Mueller, M. D. and Taylor, R. N. (2001) Immunobiology of endometriosis. Fertil. Steril. 75, 1-10.

Li, M., Li, Q., Zhao, Q., Zhang, J. and Lin, J. (2015) Luteolin improves the impaired nerve functions in diabetic neuropathy: behavioral and biochemical evidences. Int. J. Clin. Exp. Pathol. 8, 10112-10120.

Li, Y. L., Zhao, H. and Ren, X. B. (2016) Relationship of VEGF/VEGFR with immune and cancer cells: staggering or forward? Cancer Biol. Med. 13, 206-214.

Lopez-Lazaro, M. (2009) Distribution and biological activities of the flavonoid luteolin. Mini Rev. Med. Chem. 9, 31-59.

McLaren, J., Prentice, A., Charnock-Jones, D. S., Millican, S. A., Muller, K. H., Sharkey, A. M. and Smith, S. K. (1996a) Vascular endothelial growth factor is produced by peritoneal fluid macrophages in endometriosis and is regulated by ovarian steroids. $J$. Clin. Invest. 98, 482-489.

McLaren, J., Prentice, A., Charnock-Jones, D. S. and Smith, S. K. (1996b) Vascular endothelial growth factor (VEGF) concentrations are elevated in peritoneal fluid of women with endometriosis. Hum. Reprod. 11, 220-223.

Nazari, Q. A., Kume, T., Takada-Takatori, Y., Izumi, Y. and Akaike, A. (2013) Protective effect of luteolin on an oxidative-stress model induced by microinjection of sodium nitroprusside in mice. J. Pharmacol. Sci. 122, 109-117.

Park, S., Lim, W., Bazer, F. W. and Song, G. (2018) Apigenin induces ROS-dependent apoptosis and ER stress in human endometriosis cells. J. Cell. Physiol. 233, 3055-3065.

Park, S., Lim, W., You, S. and Song, G. (2019) Ameliorative effects of luteolin against endometriosis progression in vitro and in vivo. $J$. Nutr. Biochem., 67, 161-172.

Park, S. H., Ham, S., Kwon, T. H., Kim, M. S., Lee, D. H., Kang, J. W., Oh, S. R. and Yoon, D. Y. (2014) Luteolin induces cell cycle arrest and apoptosis through extrinsic and intrinsic signaling pathways in MCF-7 breast cancer cells. J. Environ. Pathol. Toxicol. Oncol., 33, 219-231.

Rana, N., Braun, D. P., House, R., Gebel, H., Rotman, C. and Dmowski, W. P. (1996) Basal and stimulated secretion of cytokines by peritoneal macrophages in women with endometriosis. Fertil. Steril. 65, 925-930.

Ryu, B., Kim, H. M., Lee, J. S., Lee, C. K., Sezirahiga, J., Woo, J. H., 
Choi, J. H. and Jang, D. S. (2016) New flavonol glucuronides from the flower buds of syzygium aromaticum (clove). J. Agric. Food Chem. 64, 3048-3053.

Bhagwat, S., Haytowitz, D. B. and Holden, J. M. (2011) USDA Database for the Flavonoid Contentof Selected Foods. Available from: http://www.ars.usda.gov/nutrientdata [cited 2017 Sep 26].

Si, H., Wyeth, R. P. and Liu, D. (2014) The flavonoid luteolin induces nitric oxide production and arterial relaxation. Eur. J. Nutr. 53, 269275.

Siristatidis, C., Nissotakis, C., Chrelias, C., lacovidou, H. and Salamalekis, E. (2006) Immunological factors and their role in the genesis and development of endometriosis. J. Obstet. Gynaecol. Res. 32 162-170.

Taylor, R. N., Ryan, I. P., Moore, E. S., Hornung, D., Shifren, J. L. and Tseng, J. F. (1997) Angiogenesis and macrophage activation in endometriosis. Ann. N. Y. Acad. Sci. 828, 194-207.

Traves, P. G., Luque, A. and Hortelano, S. (2012) Macrophages, inflammation, and tumor suppressors: ARF, a new player in the game. Mediators Inflamm. 2012, 568783.

Tsai, Y. D., Chen, H. J., Hsu, H. F., Lu, K., Liang, C. L., Liliang, P. C. Wang, K. W., Wang, H. K., Wang, C. P. and Houng, J. Y. (2013) Luteolin inhibits proliferation of human glioblastoma cells via induction of cell cycle arrest and apoptosis. J. Taiwan Inst. Chem. Eng. 44, 837-845.

Varma, R., Rollason, T., Gupta, J. K. and Maher, E. R. (2004) Endometriosis and the neoplastic process. Reproduction 127, 293-304.

Villalta, S. A., Rinaldi, C., Deng, B., Liu, G., Fedor, B. and Tidball, J. G. (2011) Interleukin-10 reduces the pathology of mdx muscular dystrophy by deactivating M1 macrophages and modulating macrophage phenotype. Hum. Mol. Genet. 20, 790-805.

Wang, D., Liu, Y., Han, J., Zai, D., Ji, M., Cheng, W., Xu, L., Yang, L., He, M., Ni, J., Cai, Z. and Yu, C. (2011) Puerarin suppresses invasion and vascularization of endometriosis tissue stimulated by 17beta-estradiol. PLOS ONE 6, e25011.

Wang, T. T., Wang, S. K., Huang, G. L. and Sun, G. J. (2012) Luteolin induced-growth inhibition and apoptosis of human esophageal squamous carcinoma cell line Eca109 cells in vitro. Asian Pac. J. Cancer Prev. 13, 5455-5461.

Wenzl, R. J. and Heinzl, H. (1998) Localization of matrix metalloproteinase-2 in uterine endometrium and ectopic implants. Gynecol. Obstet. Invest. 45, 253-257.

Wingfield, M., Macpherson, A., Healy, D. L. and Rogers, P. A. (1995) Cell proliferation is increased in the endometrium of women with endometriosis. Fertil. Steril. 64, 340-346.

Woo, J. H., Yang, Y. I., Ahn, J. H., Choi, Y. S. and Choi, J. H. (2017) Interleukin 6 secretion from alternatively activated macrophages promotes the migration of endometriotic epithelial cells. Biol. Reprod. 97, 660-670.

Wu, M. Y. and Ho, H. N. (2003) The role of cytokines in endometriosis. Am. J. Reprod. Immunol. 49, 285-296.

Zhao, H., Zhang, X., Chen, X., Li, Y., Ke, Z., Tang, T., Chai, H., Guo, A. M., Chen, $H$. and Yang, J. (2014) Isoliquiritigenin, a flavonoid from licorice, blocks M2 macrophage polarization in colitis-associated tumorigenesis through downregulating PGE2 and IL-6. Toxicol. Appl. Pharmacol. 279, 311-321. 